\title{
Sociolinguistic and Anthropological Observations in the Translation of Andrea Camilleri into Albanian
}

\author{
Diana Kastrati \\ University of Tirana \\ Faculty of Foreign Languages \\ Department of Italian
}

Doi:10.5901/jesr.2014.v4n1p525

\begin{abstract}
In modern times there is still discussion about the postulate of the untranslatability which is based on the assumption that there cannot be an authentic asymmetry between two different semantic systems. In this confrontation of school of thoughts on the efficiency, poorness, and brilliance of the translation theory and practice, fortunately, the continuous blossom of translations testifies the opposite. With the translation in albanian of Andrea Camiller, especially through some sociolinguistic and cultural anthropological observations we will try to prove the opposite of the abovementioned assumption. The translation of Camiller in albanian constitutes a fortunate encounter between the dialect invented by him in 'sicilian' and the dialectical albanian. Moreover, this case constitutes also the best confirmation that translation has historically carried out a noble mission, that of the intermediation and reconciliation of different cultures. The objective of this study is to point out the eventual problems that may arise in the translation of Camiller' novel 'La rizzagliata' (in albanian, The death of Amalia Sacerdote) through the key of sociolinguistic and anthropological reading. At the same time, we aim to highlight the favorable aspects that the albanian language has proved to have in comparison with the other foreign languages in which this author has been translated.
\end{abstract}

Keywords: culture, translatability, cultural identity, cultural anthropology, semantic systems, lexical registers, interculturality.

\section{Si può tradurre "l'intraducibile"?}

Ci imbattiamo spesso nel postulato dell'impossibilità del tradurre, ma fortunatamente la circolazione enorme dei testi tradotti di ogni genere, ci consola del contrario. Nel sistema universale della comunicazione globale il passaggio e lo scambio dei messaggi culturali, è diventato la parola d'ordine dei tempi moderni. Su questo argomento, cioè del passaggio da un sistema linguistico ad un altro e, sulla difficoltà incontratasi durante questo atto, se ne sono occupati diversi studiosi nei tempi. Secondo Whorf (1956) e Sapir (1921),"ogni lingua rappresenta una visione del mondo unica, incompatibile con ogni altro modo di percepire la realtà esterna. I parlanti sono prigionieri della loro lingua materna, incapaci di liberarsi dalle categorie e divisioni che la struttura della loro lingua impone sulle percezioni ed i pensieri". L'Ipotesi Sapir-Whorf esclude la possibilità di successo della traduzione [excludes the possibility of successful translation] (Schogt. 1986). Mentre secondo Humboldt, "ogni lingua traccia intorno al popolo cui appartiene un cerchio da cui è possibile uscire solo passando, nel medesimo istante, nel cerchio di un'altra lingua [...]una lingua straniera dovrebbe essere pertanto l'acquisizione di una nuova prospettiva nella visione del mondo fino allora vigente ... Solo perché in una lingua straniera si trasporta sempre, in misura maggiore o minore, la propria visione del mondo, anzi la visione della propria lingua, si ha la sensazione di non aver raggiunto un risultato pieno e assoluto." (Humboldt 1993). L'opinione di Humboldt al riguardo è infatti che una traduzione debba produrre degli effetti di "straniamento" nei lettori: "La traduzione ha raggiunto i suoi alti fini se ... fa sentire l'estraneo... Si distrugge la funzione del tradurre ed ogni sua utilità per la lingua e la nazione se, per avverso timore dell'inconsueto, si arriva ad evitare anche l'estraneo". (Humboldt 1993)

Attualmente possiamo dire che "Tradurre l'intraducibile", sembra un paradosso d'altri tempi. I centinaia di studi in merito, ci orientano per spunti diversi di riflessioni. In questa esposizione vorremmo fare chiarezza su un caso che appartiene alla letteratura artistica ed il cui esito (traduzione) è stato abbastanza fruttuoso. Quando si parla di traduzione letteraria, a noi traduttori ci viene in aiuto una frase di Steiner, nella sua opera Dopo Babele: "Ci sono traduzioni che non solo rappresentano la vita integrale dell'originale, ma la espongono arricchendola ed ampliandola con gli strumenti operativi della propria lingua. [...] anche se si tratti di un caso assoluttamente straordinario, ci sono traduzioni le quali rieintegrano e riescono a creare un equilibrio ed una stabilità radicale di parità tra due opere, due lingue, due comunità di esperienza storica e sensibilità contemporanee". Ma quante siano le reali possibilità che un traduttore possa riconoscersi 
in questa definizione lapidaria di Steiner? Quale possa essere quello strumento onnipotente che porti il traduttore ad ottenere quella pulsazione di una ricreazione come lo è la traduzione? Come si possano riportare nella lingua d'arrivo le tonalità della lingua di partenza?

Noi ci soffermeremo ad analizzare il caso della traduzione di uno scrittore che nella letteratura italiana del secolo scorso e di questo appena iniziato, rappresenta infatti un fenomeno oseremo dire, unico. Si tratta di Andrea Camilleri. L'opera "La rizzagliata" (in albanese, Vdekja e Amalia Saçerdotes) presa in esame in questa sede, si tradusse in albanese nel 2011 e fu pubblicato nel 2012. Oramai il nome di Camilleri si assoccia con la parola "fenomeno", giusto il camillerismo. Da decadi lui gode lo status di pochi privilegiati tra la schiera degli scrittori contemporanei vivi, quello del conoscersi in tutto il mondo, a prescindere dal fatto che scriva in dialetto. Tradotto nella maggior parte delle lingue del mondo (più di sessanta), ha raggiunto l'apice del successo anche in paesi (come Germania, Finlanda, Giappone, Corea, Arabia saudita ecc), le cui lingue non hanno nessun punto di contatto con la famiglia dell'italiano, ma soprattutto con il contesto culturale in cui l'autore situa i suoi romanzi. Ė riconosciuto da tutti il fatto che lui scrive in un dialetto reinventato, una specie di mix tra il siculo e l'italiano standard. Lui dice: "La lingua che uso nei miei libri non è la trascrizione del dialetto siciliano. Ė una reinvenzione del dialetto ed è il recupero di una certa quantità di parole contadine, che si sono perse nel tempo. Cataminarisi ("muoversi"), per esempio, non viene adoperata nel linguaggio piccolo borghese che era il nostro: era linguaggio contadino." È fuori dubbio che proprio in questo tipo di scrittura debba essere letto il successo internazionale che ha scosso Camilleri. La freschezza della sua scrittura sta proprio nella sua capacità creativa unica per offrirti un panoramo seducente ed intrigante della Sicilia, della sua terra nativa, come nessun altro prima di lui.

La patente che Camilleri con la sua lingua, diede alla sua comunità magica.

Non è un fenomeno isolato che scrittori imponenti della letteratura mondiale ammirata dai lettori di tutto il globo, della letteratura novità, della letteratura avangarde, abbiano creato una realtà magica nelle loro opere. Detto diversamente, che l'opera sia una realtà magica di per sé, un piccolo laboratorio sperimentale fatto di materiale umano, ma in condizioni molto specifiche. Il primo nella lista spicca il nome di Marques; la realtà è sua, il messaggio universale. Lo stesso per il Camilleri il quale si spinge oltre questo confine. Lui è riuscito a presentare al mondo intero l'unica realtà che conosce perfettamente, il microuniverso dei paesini di Sicilia (la Vigata immaginaria) che, se non fosse per lui, nemmeno l'Italia non sarebbe tanto sicura che lo portasse dentro il suo territorio. Non la inventò, gliela presentò, però in un modo autentico: conferendole la sua sigla distintiva, patentandola con la sua lingua caratteristica. Di conseguenza, rese indissolubile l'essenza con la forma, identitificando l'una con l'altra. Ciò costituisce la possibile deduzione del suo universalismo attraverso il fenomeno novità: il camilleranismo. Alla legittima domanda dove stia la chiave del suo successo mondiale, lui risponde dicendo: perché penso in siciliano! Questa fu la scoperta della sua maturità: pensare in dialetto. Lui diventò il traduttore simultaneo di se stesso. Dal Piemonte in Sardegna, dalla Lombardia in Calabria, dal Veneto in Puglia, i lettori gustano la dimensione universale dell'autore siculo senza inciampare nei "trannelli" linguistici e culturali della sicilianità. Tale dimensione si estende fino ad abbracciare il piacere del lettore swedese, finlandese, tedesco, giapponese e, anche di quello albanese. Che cosa fa di lui uno scrittore ammirato anche in Giappone o nei paesi nordici, culture assoluttamente distanti e diverse dalla sua? Come dobbiamo capire il fenomeno linguistico ed artistico di Camilleri? Lui dice che la sua salvezza è il dialetto. "Quando il discorso si fa serio, usa il dialetto!". Dal momento che ha intrapreso la strada del dialetto nella sua creatività artistica, tutto si è illuminato come per d'incanto.

\section{La stratificazione delle varietà sociolinguistiche di Camilleri.}

Le varietà linguistiche più usate da Camilleri, sono almeno cinque, ciascuna con una sua precisa funzione stilistica. Eccole in rassegna:

\section{La varietà mista}

Vizmuller-Zocco (2004) in II caso Camilleri, parla appunto di un code mixing, cioè una collocazione equilibrata dei codici. Tale varietà trova applicazione nei casi di sfoghi emotivi dei personaggi. In seguito il dialetto siciliano viene a reintegrarsi con la lingua italiana standard. Interessante risulta il fatto che Camilleri spesso attribuisce allo stesso personaggio un misto di varietà, a seconda della situazione comunicativa. Concretamente, Michele (il protagonista del romanzo in questione) ne fa un uso regolare dello standard dentro gli uffici della RAI, ma nello scontro con dei dipendenti non tanto "simpatici" sfoggia un dialetto per niente elegante. L'italianizzazione del linguaggio si realizza chiaramente usando morfemi italiani i quali vengono attaccati ad una base lessicale siciliana. Le basi si scelgono dallo stesso autore. L'uso del dialetto si evidenzia tramite l'interferenza dello standard in alcuni passaggi i quali vengono attribuiti ai personaggi o 
eventi che rientrano in ambiti pubblici, come l'istituzione della televisione statale (RAI), oppure dentro il Tribunale ecc. Tale fenomeno si chiama code switching, ossia, lo scambio dei codici linguistici. (D'Agostino 2007).

\section{L'Ibridazione}

Oppure la fusione dei due sistemi linguistici. Questo tipo di meccanismo accentua ulteriormente l'aspetto creativo dell'autore. Tale fenomeno include solo alcune parole, non tutto il linguaggio, dunque prevede la fusione completa delle regole e delle unità linguistiche di due ligue diverse. Le barriere linguistiche spariscono dal momento che gli elementi stilistici vengono combinati nei modi dai più imprevedibili, creando in questo modo delle parole totalmente nuove. P.es.: fatigazza - un incrocio con la faticaccia (termine dello standard); in albanese: lodhje e madhe, këputje; travaglio travagghiu, incrociata con la parola lavoro (termine dello standard); in albanese: punë e mundimshme; quanno - quannu iincrociato con la parola quando (termine dello standard); in albanese: atëherë kur; vrigognuse - vrigognusi, incrociato con la parola vergognose (termine dello standard); in albanese: i turpshëm, i ndrojtur. Quest'ultima trova la sua perfetta equivalente nel dialetto gege dell'albanese: i marrshëm, la quale ha una carica stilistica molto spiccata, forse più del termine dello standard.

\section{L'italiano elaborato scritto e parlato \\ 4. L'italiano popolare,}

attribuito maggiormente ai personaggi non colti,caratterizzato da modifiche come conseguenza dell'unione con dei dialettalismi, non correttoe ortograficamente parlando, con un uso inopportuno dei verbi, dei pronomi e degli articoli.

\section{II paradialetto,}

Ossia la libera scelta artistica di Camilleri, autorizzandosi di usare delle forme mai udite e che non rientrano in nessuna classificazione sociolinguistica.

La rizzagliata è scritto quasi in 90\% in dialetto siciliano-camilleriano. Da lettore o da studioso si rimane inchiodati ed incantati dall'uso di una lingua così complessa, fatta di tanti strati e rivestita di connotazioni regionali. Grazie a questo meccanismo così ben riuscito, l'autore è riuscito a rendere tutte le sfumature mentali, spirituali e sociali dei siciliani a cominciare dal loro tragismo proverbiale, all'ironia, alla teatralità che veste alcuni personaggi, al senso dell'umor, all'aspetto erotico. II dialetto e tutte le forme sopraesposte, sono l'essenza stessa dell'opera.

\section{II traduttore camilleriano e le sue caratteristiche operative}

Vediamo quali siano stati i requisiti indispensabili del ri-creatore dell'opera, ossia del traduttore (in albanese 0 in altre lingue) di un autore come Camilleri:

- $\quad$ Per le peculiariatà che la traduzione letteraria rappresenta e, in fattispecie per la scrittura in un dialetto quasi inventato del Maestro, i requisiti indispensabili che debba avere il traduttore sono tanti: le ottime competenze linguistiche delle due lingue, quella di partenza e quella d'arrivo, conoscenza della tipologia testuale e del trama trattato, esperienza pratica, sensibilità linguistica nella LP e nella LA, intuizione come elemento imprescendibile per un'alta qualità del prodotto, sensibilità artistica. In questo caso che stiamo esaminando, tutto il pacchetto sopracitato resta in vigore, ma l'aspetto più interessante si rivela nell'uso dei fattori extralinguistici come chiave di lettura psichica ed interpretativa dell'autore e del testo originale, tra cui la conoscenza dell'antropologia culturale della bella isola.

- Inoltre, la traduzione in albanese presupponeva, a mio avviso, un coinvolgimento umano in maniera diretta e non mediata, diventando in tal modo una cassa di risonanze dell'emotività dell'autore e degli eventi. Il risultato è un processo di trasferimento ed interpretazione non solamente dei segni, ma bensì della vita con vita.

- $\quad$ Di conseguenza, spuntava fuori anche una situazione inevitabile di empatia da parte del traduttore. Infatti l'empatia "non è una sensazione, né un sentimento, né un atto della percezione interna di sé, e tanto meno è riconducibile al ricordo e all'immaginazione, ma è atto concreto e originario, attraverso il quale possiamo cogliere in modo non-originario un vissuto straniero" (Stein 2009). Altro non è che quella capacità di abitare nello stesso modo in due mondi diversi. In questo senso, l'empatia rappresenta anche "la modalità mediante la quale raccogliamo dati psicologici a proposito delle altre persone e, quando esse dicono ciò che pensano o sentono, noi immaginiamo la loro 
esperienza interna...Mediante l'empatia noi miriamo a discernere, in un singolo atto di riconoscimento certo, configurazioni psicologiche complesse, che altrimenti potremmo definire mediante la laboriosa presentazione di una serie di dettagli, o che potrebbero addirittura collocarsi al di là delle nostre capacità di definizione" (Kohut 2003). Dunque, il tradutore diventa una specie di "filtro umano" nel passaggio del messaggio da un sistema culturale e cognitivo in un altro.

- L L'esistenza dell'aspetto "psichico" nella traduzione, cioè quello che si collega con l'interpretazione del messaggio. Questo processo consiste nella decostruzione a volte conscia, a volte no, del testo originale per passare poi nella "riformulazione" che porta infine all'interpretazione ed alla traduzione.

- A pari passo ci va l'operazione minuziosa del traduttore nell'identificare le varietà linguistiche e sociolinguistiche della lingua di partenza da adottare nella LA. Una volta compiuta la scelta, rimane solo l'attuazione a seconda le situazioni comunicative ed il profilo dei personaggi. Ricordiamo qui p.es. il personaggio di Lamantia, (l'informatore di Michele, il protagonista del romanzo), il quale nel "pieno delle regole" della tipologia sociale a cui appartiene, gli viene attribuito un lessico volgare: "...la povera ragazza...la fanciulla stroncata nel fiore dell'età....be, tanto bocciuolo non era dato che era sbocciata scopanno regolarmente con lo zitto?" (Camilleri 2009). Ovviamente, in questo caso l'equivalenza diretta, stilisticamente parlando, non è sufficiente, per cui il traduttore si serve del metodo della compensazione rendendo molto meglio il profilo del personaggio. Infatti, nella traduzione in albanese troviamo: "E shkreta vajzë...nji gonxhe qi s'e lanë të lulëzonte...aq gonxhe nuk ishte, se kishte shperthye kaherë, qysh se shkërdhehej rregullisht me të fejuemin?" (Camilleri 2012)

\section{L'albanese "favoreggiato" vs le altre lingue straniere}

Tradurre Camilleri significa una vera battaglia. La prima cosa che ti salta agli occhi e che debba essere rispettata da qualsiasi traduttore, è appunto la presenza della stratificazione dei registri e degli stili di cui ne parlammo sopra. In rispetto di questo fenomeno, necessitava che si chiamasse in aiuto una variante dialettale albanese che poteva rendere meglio tutte quelle sottilezze linguistiche, ma soprattutto che rispecchiassero ottimamente la costituzione psico-sociale siciliana. L'albanese comprende essenzialmente due dialetti: il gegë, che coincide con la lingua del nord d'Albania, e il toskë, quello del sud. Optai per l'uso della variante cittadina gegë nei dialoghi (che costituiscono $70 \%$ dell'opera), mentre il resto, cioè la parte narrante in lingua standard. Quest'ultima risultava insufficiente e sbiadita di fronte alla galleria linguistica e stilistica multicolore dell'opera in originale. Non fu una scelta casuale: per ragioni puramente stilistiche ma, soprattutto, per ragioni antropologiche, la zona del nord trova punti in comune con i siciliani e "la sicilianità". Ecco alcuni di questi fattori "benevoli" e favoreggianti dell'albanese durante la traduzione dell'opera di Camilleri:

- A differenza dei tentativi estremi professionali e scrupolosi compiuti dagli altri traduttori di Camilleri in altri paesi, i quali hanno scelto appunto l'uso di un dialetto del proprio paese, la traduttrice albanese era favoreggiata al massimo dovuto primariamente ad una circostanza extra- linguistica; si tratta della dimensione socio-culturale-antropologica dei siciliani che ha molte affinità e punti di contatto con la dimensione antropologica degli albanesi. Indipendentemente dalla buona volontà che caratterizza normalmente qualsiasi traduttore quando inzia un'impresa del genere, non sempre la comunicazione e la traducibilittà possa essere realizzata efficentemenete. Tanti concetti sono intimamente legati alla cultura del paese d'origine dell'opera, che forse non si possano rendere tramite un altro codice linguistico, danneggiando seriamente, di conseguenza, la realizzazione dell'operazione. Ciascun popolo vede la realtà in modo differente dall'altro, influenzato potentemente dall'ambiente e dal proprio percorso storico. Secondo il filosofo e linguista tedesco Wilhelm von Humboldt, ogni lingua è causa e riflesso del Weltanschaung, meglio dire della propria visione per il mondo in cui vive e per la propria cultura del vivere. Dunque, la diversità linguistica non è altro che un disaccordo in merito alle visioni sul mondo. In questo ambito, Humboldt, trattando la questione della traduzione dice che l'intento di quest'ultima è proprio quello di permettere a captare la visione differente del mondo e dello spirito della cultura presso cui si è tessuto il testo originale. Detto diversamente, si tratta di quello che noi lo chiamiamo "respiro" dell'opera letteraria, il suo "ésprit". Nel caso del rapporto del dialetto siciliano di Camilleri con l'albanese dialettale, per fattori incondizionati da noi, questo respiro risulta interamente afferrabile, tanto da gustarlo in pieno.

- $\quad$ Di conseguenza, le circostanze socio-politiche-culturali siciliane che per un traduttore tedesco o nordeuropeo erano del tutto ignote, forse anche inconcepibili, per noi/me, erano familiari. In tal caso, purtroppo, il comune denominatore del sistema di corruzione che costituisce una legge per l'isola di Sicilia, ma tanto quanto anche in Albania, mi aiutò a decifrare e decodificare anche le più sottili sfumature dei "trucchi" politici, gli invisibili meccanismi del malfunzionamento "meditarraneo" che trovano una stratificazione comune nella costituzione psico-sociale dei due 
rispettivi paesi. La traduzione in questo caso diventava un "dejà vu".

- Rimanendo in questa ottica socio-antropologica, l'uso da parte mia del dialetto scutarino (della città di Scutari, capoluogo del Nord d'Albania) cittadino che coincide anche con la mia origine, costituiva la chiave per apririmi la strada verso il contesto siciliano il quale coincideva perfettamente con quello della realtà della comunità di questa città. Tali somiglianze si possono notare nella divisione ben scandita dei ceti sociali a Scutari come a Palermo, nell'immobilità della gerarchia sociale di ambedue le realtà, nelle tradizioni familiari, nelle mentalità urbane e della periferia relative dei due centri.

- $\quad$ Per certi tratti, segmenti sintattici, il dialetto siciliano trova punti di contatto con la struttura dialettale scutarina. Un esempio lampante costituisce l'uso del verbo alla fine della frase nel caso di un registro informale scorretto. Per esempio: "Cca' sugno",in albanese: "Këtu jam", oppure "Troppo presto venisti", in albanese: "Si shumë heret ke ardhë!". L'uso di una forma del genere, assieme ad altre forme dialettali tipiche siciliane e scutarine, trovavano la naturalezza e la scioltezza nei dialoghi, rendendo la comunicazione molto più scorrevole e disinvolta. Aggiungiamo il fatto che la scrittura di Camilleri ha molti aspetti cinematografici. Questo suo tratto essenziale, il quale viene a completarsi con le mezze proposizioni dei personaaggi non colti che accellerano il passaggio delle "immagini-scene" con le quali Camilleri muove la sua "camera"=penna, facilitavano la stesura traduttiva.

- II favoreggiamento che l'albanese dialettale ha nei confronti delle altre lingue straniere, consiste anche in un altro aspetto: quello del linguaggio erotico, molto frequente nelle opere di Camilleri. Suscita interesse per esempio, conoscere i tentativi immani che ha fatto il traduttore tedesco per rendere l'intensità delle scene erotiche del romanzo, in modo da avvicinarsi al colorito originale degli isolani. Moshe Kahn, il nome del traduttore tedesco, dichiara che ha rinunciato volontariamente alla scelta di un dialetto tedesco per adottarlo alla traduzione delle scene erotiche. Per questa ragione lui si è rivolto allo stile barocco della scrittura per poter trovare un equivalente idoneo alla passionalità e ricchezza lessicale sicula. In una lettera che ha scritto a Camilleri, lui dice:

"Alla fine vi voglio ancora parlare brevemente di un problema che mi tortura in grande stile appena quello si presenta, cioè il linguaggio erotico di cui abbonda anche la scrittura di Camilleri. Ah, Andrea, se sapessi davanti a quale abbisso mi stai mettendo!

Perché tu non ti rendi conto del fatto che la lingua tedesca ha un linguaggio erotico molto ristretto e quel poco è ambientato per la maggior parte, se lo vogliamo localizzare anatomicamente per la sua provenienza, nella zona del deretano, mentre la lingua italiana - come tutte le lingue romanze - gioca liberamente, gioisamente e impudicamente con le parti sessuali. Perché una cosa è di fondamentale importanza: mai una espressione o addirittura una frase erotica deve avere il sapore del tradotto, deve invece apparire del tutto naturale, come tutta la traduzione, come se il traduttore l'avesse trovata in altri strati del linguaggio quotidiano non più conosciuti. In fondo è sempre una nuova operazione, $i$ risultati trovati una volta, in una determinata circostanza per un determinato libro, non sono necessariamente repetibili o trasferibili a un altro libro. Vi posso assicurare questo: ho impiegato infinitamente tanto tempo per questo trapianto del deretano erotico-linguistico del tedesco verso le parti sessuali anteriori del linguaggio italiano... e credo di potere affermare che io, con la mia operazione, sia riuscito ad arricchire il tedesco erotico considerevolmente." (Kahn 2004)

- Lessicalmente parlando, a differenza del tedesco, che è una lingua molto riservata e parsimoniosa nella terminologia erotica, l'albanese è molto ricco e variegato. Essendo l'Albania una zona "calda" del Mediterraneo, è logico che anche la lingua ne rispecchia questo aspetto con una bella ricchezza di termini e visioni, stando così con molta "dignità" accanto al dialetto siciliano. L'argomento del sesso nel linguaggio popolare è un elemento fondamentale. Lo stesso si può dire della passionalità balcanica-mediterranea che caratterizza la popolazione albanese, tratto che agevolisce ulteriormente la traduzione. Però, quello che sembra essere un punto di vantaggio per la traduzione, ha anche l'altra faccia della medaglia. Nella posizione della traduttrice e di donna, mi sono sentita diverse volte in dilemma davanti alla marea delle frasi tipicamente siciliane e che vengono ripetute con una frequenza vertiginosa. II mio dilemma aveva a che fare con il rapporto etica-bellezza artistica. Dunque, la famosa frase "che minchia vuo' la quale non ha la stessa frequenza d'uso in albanese, doveva essere tradotta con la giusta equivalente "salata", o si doveva moderare il termine? Si doveva rispettare quel "respiro" di cui parlavamo prima, o la parola si doveva interrompere con i puntini di reticenza? Lo spunto verso una carica stilistica forte, mi spinse ad optare per la prima. Mi sembrava scorretto non usare quella parola che così tanto dava al linguaggio.

II profilo di Andrea Camilleri come il Maestro delle lettere, suscita curiosità ed incanto. L'atmosfera dei suoi romanzi respira la bellezza dell'isola misteriosa, segna i sensi di chi lo legge. La traduzione delle sue opere costituisce la miglior conferma che questo atto di trasferimento da un sistema semantico linguistico in un altro, rimane uno dei più efficaci mediatori di concilazione di tutte le culture, storicamente. 


\section{Bibliografia}

Camilleri, A.(2009). La rizzagliata, Sellerio Editore Palermo.

Citarrella, C. (2010), Tradurre le metafore. Appunti delle lezioni, Compostampa, Palermo.

Colli, G. (1979). Al di là del bene e del male, Adelphi.

Eco, U. (2003). Dire quasi la stessa cosa. Esperienze di traduzione, Bompiani.

D’Agostino, M. (2007). Sociolinguistica dell'Italia contemporanea, il Mulino.

Kohut, H. (2003). Introspezione ed empatia, Bollati Boringhieri.

Ricoeur, P. (2008). Tradurre l'intraducibile/ Sulla traduzione, In dialogo 2, Urbaniana University Press.

Salmon, L. (2003). Teoria della traduzione. Storia, scienza, professione, Vallardi Editore, Milano.

Scarpa, F. (2008). La traduzione specializzata, un approccio didattico professionale, Editore U. Hoepli, Milano.

Sorgi, Marcello. (2000). La testa ci fa dire - Dialogo con Andrea Camilleri, Palermo, Sellerio Editore.

Steiner.G. (1994). Dopo Babele. Aspetti del linguaggio e della traduzione, Garzanti, Milano.

Stein, E. (2009). II problema dell'empatia, ed.Studium, Roma.

Vizmuller-Zocco, J. (2004). II caso Camilleri. Letteratura e storia, Sellerio Editore, Palermo.

http://www.andreacamilleri.net/camilleri/linguaggio.html13 